\title{
Does the No Alternatives Argument need Gerrymandering to Be Significant?
}

\author{
Richard Dawid
}

February 18, 2021

\begin{abstract}
In a recent paper, Tushar Menon has argued that the noalternatives argument can only be significant if the priors for numbers of alternatives are tuned in an implausible way (gerrymandered, as he calls it). In this paper, I demonstrate that priors needed for making a no-alternatives argument significant are in line with what can be plausibly assumed in a successful research field.
\end{abstract}

\section{Introduction}

Meta-empirical theory confirmation is a strategy to assess the probability of a theory's viability based on assessing the spectrum of possible alternatives to that theory (Dawid 2006, 2013). If one comes to the conclusion that the number of possible theories is probably very small or even one, this assessment generates a substantial amount of trust in the theory at hand. In Dawid (2013), three arguments of meta-empirical confirmation have been discussed. ${ }^{1}$ One of them, the no-alternatives argument (NAA), was formally shown to amount to confirmation under very plausible conditions in (Dawid et al. 2015). In a recent paper, Menon (2019) argues that the confirmation provided by the NAA can only be significant if one assumes a set of priors for the numbers of possible theories that is carefully tuned (gerrymandered, as Menon calls it) to achieve that goal. Menon argues that the required priors do not constitute natural choices, which renders the NAA largely ineffective under typical scientific circumstances.

\footnotetext{
${ }^{1}$ In Dawid (2013), they are presented as examples of a wider group of arguments of non-empirical theory assessment. The three arguments that are based on assessing the spectrum of alternatives have been given the name "meta-empirical confirmation" (MEC) in Dawid (2020).
} 
The present article demonstrates that the priors required for making the NAA significant can be extracted in an empirically successful research field from a second argument of MEC, the meta inductive argument (MIA).

\section{The No-Alternatives Argument and Menon's Criticism}

The no-alternatives argument (NAA) aims to significantly increase trust in the viability of a theory $H$ that accounts for data $E_{0}$ in contexts where core predictions of $H$ have not yet been empirically confirmed. The argument is based on the observation $F_{N A}^{H}$ that, despite considerable efforts, no alternative theory to $H$ that can account for $E_{0}$ has been found. From this observation, it is inferred that there probably are no or very few alternatives to theory $H$. The assessment that the number of alternatives is probably very small in turn leads to increased trust in the viability of $H^{2}$

Dawid et al. (2015) give a proof that NAA indeed provides confirmation under plausible conditions. Let $T^{H}$ be the proposition that hypothesis $H$ is empirically viable within a certain empirical horizon ${ }^{3}$ and let $Y_{i}^{H}$ denote the propositions that there are $i$ scientific theories that can account for the data $E_{0}$ that is accounted for by theory $H$. If five mild and plausible conditions are fulfilled, $F_{N A}^{H}$ provides Bayesian confirmation of $H$ :

$$
P\left(T^{H} \mid F_{N A}^{H}\right)>P\left(T^{H}\right)
$$

Then five conditions are (for their full presentation and explanation see Dawid et al. 2015, pp. 218-19):

A1. A2. Conditions on the conditional independence of variables.

A3. The conditional probabilities

$$
P\left(\mathrm{~F}_{\mathrm{NA}}^{\mathrm{H}} \mid \mathrm{Y}_{\mathrm{i}}^{\mathrm{H}}\right)
$$

are non-increasing in $i^{4}$

\footnotetext{
${ }^{2}$ This brief characterization of NAA leaves out a number of subtleties that need to be addressed in order to make the argument fully coherent and meaningful. The reader interested in a full discussion is referred to Dawid (2013) and Dawid et al. (2015).

${ }^{3}$ I call a theory empirically viable within a given empirical horizon if it can account for all empirical data that can be collected within that horizon (for example, up to a given energy scale).

${ }^{4}$ For simplicity reasons, I omit the variable $D$ that controls the complexity of the problem in the full formulation of condition A3.. The formal specification of $D$ will play no role in the further analysis in this paper.
} 
A4. The conditional probabilities

$$
P\left(\mathrm{~T} \mid \mathrm{Y}_{\mathrm{i}}^{\mathrm{H}}\right)
$$

are non-increasing in $i$.

A5. There is at least one pair $(i, j)$ with $i<j$ for which

(i) $P\left(\mathrm{Y}_{\mathrm{i}}^{\mathrm{H}}\right) P\left(\mathrm{Y}_{\mathrm{j}}^{\mathrm{H}}\right)>0$,

(ii) $P\left(\mathrm{~F}_{\mathrm{NA}}^{\mathrm{H}} \mid \mathrm{Yi}^{\mathrm{H}}\right)>P\left(\mathrm{~F}_{\mathrm{NA}}^{\mathrm{H}} \mid \mathrm{Y}_{\mathrm{j}}^{\mathrm{H}}\right)$ for some $j \in \mathbb{N}$, and

(iii) $P\left(\mathrm{~T} \mid \mathrm{Y}_{\mathrm{i}}\right)>P\left(\mathrm{~T} \mid \mathrm{Y}_{\mathrm{j}}\right)$.

The proof given by Dawid et al. (2015) shows that NAA provides confirmation but does not establish the significance of that confirmation. Confirmation could be marginal and therefore, though formally realized, unhelpful for scientific theory assessment. This situation is in agreement with the claim made in Dawid (2013) that modes of meta-empirical confirmation achieve significance only in conjunction.

Menon now raises the question as to how natural and plausible significant confirmation would be in the scenario Dawid et al. (2015) describe. He does not apply a rigid threshold for the significance of confirmation but requires that $P\left(T^{H} \mid F_{N A}^{H}\right)-P\left(T^{H}\right)$ be a "non-negligible number". He reaches the conclusion that significant confirmation in this sense can only be achieved by NAA based on deliberately chosen sets of priors for three important kinds of variables.

The required priors amount to a considerable sharpening of the conditions A3-A5 spelled out above. Rather than the very soft A3-A5, significant confirmation would require the following much stronger conditions:

SC3 $P\left(\mathrm{~F}_{\mathrm{NA}}^{\mathrm{H}} \mid \mathrm{Y}_{\mathrm{i}}^{\mathrm{H}}\right)$ must show a significant decrease in $i$ for low $i$-s.

SC4 $P\left(\mathrm{~T}^{\mathrm{H}} \mid \mathrm{Y}_{\mathrm{i}}^{\mathrm{H}}\right)$ must show a significant decrease in $i$ for low $i$-s.

SC5 $P\left(Y_{i}^{H}\right)$-s must have high priors for very low $i$-s that quickly fall off for higher $i$-s.

Menon argues that SC5, in particular, is problematic. It is not a generic choice of priors but would amount to what he calls "gerrymandering": specific factually unsupported prior assumptions are made just in order to allow for significant confirmation based on NAA. Gerrymandering of this kind would make NAA clearly question-begging. Moreover, Menon argues, even if 
there were good reasons for assuming high probabilities for low $i$-s as priors, NAA would be in trouble. Given that high probabilities for low numbers of alternatives are exactly what NAA is supposed to establish, if those high probabilities can be established already before NAA gets started, NAA seems superfluous.

\section{The Meta-Inductive Argument}

Menon's formal analysis is fully accurate. The distribution of priors he singles out is of the kind one indeed needs for significant NAA. What I will contest in the following is his verdict that this choice of priors is gerrymandered in scientific contexts where NAA is taken seriously by scientists.

The crux of the matter boils down to the fact that NAA requires support from other forms of meta-empirical confirmation to get off the ground (Dawid 2013, Section 3.1.). In the absence of such support, NAA indeed faces the problem Menon describes. Scientific reasoning in a mature scientific field, however, is distinguished by the fact that NAA can be supported by another MEC argument, the meta inductive argument (MIA). I will set out to show that MIA in a predictively successful research field very plausibly generates the distribution of probabilities that is demanded by Menon. These probabilities, which constitute posteriors with regard to updating under MIA, then can serve as priors in a NAA argument.

Let me briefly recapitulate the general structure of MIA. The argument starts from the observation that theories that satisfy some set of conditions $K$ tend to be predictively successful once empirically tested. Based on that observation, MIA suggests that a new theory that also satisfies $K$ has a significant chance of being predictively successful as well. Being an instantiation of MEC, the inference can be framed in terms of an assessment of the spectrum of possible (conceived and unconceived) alternative theories. MIA on that understanding establishes that the number of possible theories is probably very limited, maybe even limited to one single theory.

The role of conditions $K$ is to specify a group of scientific theories that have a clear tendency of predictive success. Obviously, the history of science knows a vast number of scientific theories that lack predictive success. Therefore, conditions $K$ must go beyond the mere scientificality conditions a theory needs to meet in order to count as an alternative theory in a MEC analysis. $K$ should for example include conditions that constrain the analysis to a specific research field or subfield where predictive success seems conspicuous. Moreover, it seems helpful to admit only theories that were developed after a certain point in time. 
These two types of conditions still won't suffice for establishing a clear tendency of predictive success in most cases, however: next to every research field, past or present, generates many candidate theories that are not much believed in and never find empirical support. In scientific contexts where many scientific theories with a wide spectrum of mutually inconsistent predictions can be developed, most of those theories fail. Therefore, it is important to add to $K$ a condition that selects only those theories to which a NAA applies. This is in line with the mechanism of MIA because a NAA itself indicates predictive success by establishing constraints on the spectrum of possible alternatives. Considering only theories that had no known alternatives before being tested is a powerful tool for isolating a group of theories with a high rate of predictive success. Further criteria could be added to $K$, but we will assume only the three criteria introduced above.

The question we now want to address is the following. Does MIA provide the basis for assuming the set of probabilities Menon establishes as a necessary precondition for having significant confirmation based on NAA?

\section{Meeting the First Two Conditions}

Let us first address the requirements SC3 and SC4. Condition SC4 requires that $P\left(\mathrm{~T}^{\mathrm{H}} \mid \mathrm{Y}_{\mathrm{i}}^{\mathrm{H}}\right)$ must show a significant decrease in $i$ for low $i$-s. This condition is generally assumed to be met when representing MEC.

In models of MEC reasoning, theories are individuated based on their distinct predictions within a given empirical horizon. Once all data that can be collected within that empirical horizon is in, all but one of the theories that can possibly be developed in agreement with $E^{0}$ are ruled out. On this basis, one assumes that developing a theory in accordance with the available data $E^{0}$ resembles making a random pick from those theories that are consistent with $E^{0}$. In other words, before collecting further data $E$, one has no reason to assume that the theory that is predictively successful with respect to $E$ is easier or less easy to find than any other theory that is consistent with $E^{0}$. This assumption implies the following dependence of the chances of novel confirmation on the number of possible alternatives:

$$
P\left(T^{H} \mid Y_{i}^{H}\right)=1 / i
$$

The calculations in this paper will be based on (4). But even if one takes Equation (4) to be an idealization, a considerably weaker relation that preserves the basic view on theory development reflected in Equation (4) - 
such as replacing $1 / i$ by $i^{-x}$ with $\mathrm{x}$ smaller than one but significantly higher than zero - would be sufficient for fulfilling condition SC4.

Condition $\mathrm{SC} 3$ requires that $P\left(\mathrm{~F}_{\mathrm{NA}}^{\mathrm{H}} \mid \mathrm{Y}_{\mathrm{i}}^{\mathrm{H}}\right)$ must show a significant decrease in $i$ for low $i$-s. This condition looks a priori plausible. In a context where many alternative theories can be built, it seems plausible to expect that, if one of those theories has been found, others can be found as well. If there is just one possible theory, obviously no others can be found. And if the few possible theories exist, the more plausible it seems that complications related to the construction of all but one of them have prevented the construction of any of the other theories so far.

Condition SC3 is further supported by any case of successful MIA reasoning that involves the lack of known alternatives to a given theory as an element of conditions $K$. If a strong MIA cannot be established based on all theories in a research field but can be established once the condition that no alternatives are known is applied, this establishes, based on successful MEC reasoning, that there is a significant correlation between the number of theories that are discovered and the number of alternative theories that exist. Without that correlation, it would not be explicable why requiring the lack of discovered alternatives was helpful for generating a successful MIA.

This leaves condition SC5, which to justify is the main aim of this paper. As pointed out above, we need to address two questions in that context: First, does MIA generate a set of $Y_{i \text {-S }}$ of the kind Menon shows to be required for having a significant NAA? Second, if it does, why do we need NAA at all as an independent argument, rather than merely using the condition that a theory has no known alternatives as one of the conditions $K$ ?

We will approach the answer step by step by first calculating a simplified scenario and then backing up its results based on a more general probabilistic analysis.

\section{Updating on the Success of an Individual Theory}

Let us first look at one individual case of predictive success of a theory $H_{1}$. Consider a theory $H_{1}$ that has been developed based on a data set $E^{0}$ and successfully predicts data $E$. Let $T^{H_{1}}$ denote the empirical viability of $H_{1}$ with respect to data $E .^{5}$ Let $Y_{i}^{H_{1}}$ be the statement that $H_{1}$ is one of $i$ possible theories that can formulated in agreement with data $E^{0}$. The question we

\footnotetext{
${ }^{5}$ In other words, we assume conclusive confirmation of the theory $H_{1}$ within the empirical horizon that covers data $E$.
} 
want to ask is the following: if we start with a given set of priors $P\left(Y_{i}^{H_{1}}\right)$, what are the posteriors $P\left(Y_{i}^{H_{1}} \mid T^{H_{1}}\right)$ ?

Let us now introduce the following choice of priors for $Y_{i}^{H_{1}}$ :

$$
\begin{array}{rrr}
P\left(Y_{i}^{H_{1}}\right)=1 /\left(\sum_{j=1}^{N} j^{-1}\right) i & \forall & i \leq N \\
P\left(Y_{i}^{H_{1}}\right)=0 & \forall & i>N .
\end{array}
$$

For this choice of priors, we have $P\left(Y_{i}^{H_{1}}\right) \rightarrow 0$ for all $i$ in the limit $N \rightarrow \infty$. In this limit, the expectation value for the number of alternatives $i$ goes to infinity. Still, the priors favor individual small number for $i$ over individual large numbers. We will motivate this choice of priors in a wider context in the next section. Writing the total probability for $P\left(T^{H^{1}}\right)$, Equation (4) gives:

$$
P\left(T^{H_{1}}\right)=\sum_{i=1}^{\infty}\left(P\left(Y_{i}^{H_{1}}\right) P\left(T^{H_{1}} \mid Y_{i}^{H_{1}}\right)\right)=\sum_{i=1}^{\infty} i^{-1} P\left(\mathrm{Y}_{\mathrm{i}}^{\mathrm{H}_{1}}\right)
$$

Equations (5), (6) and Bayes' theorem then lead to

$$
\begin{aligned}
P\left(Y_{i}^{H_{1}} \mid T^{H_{1}}\right) & =\frac{P\left(T^{H_{1}} \mid Y_{i}^{H_{1}}\right) P\left(Y_{i}^{H_{1}}\right)}{P\left(T^{H_{1}}\right)} \\
& =\left(i^{-2}\right)\left(\sum_{j=1}^{N} j^{-1}\right)^{-1}\left(\left(\sum_{k=1}^{N} k^{-2}\right)\left(\sum_{j=1}^{N} j^{-1}\right)^{-1}\right)^{-1} \\
& =\left(i^{-2}\right)\left(\sum_{j=1}^{N} j^{-2}\right)^{-1} \\
\lim _{N \rightarrow \infty} P\left(Y_{i}^{H_{1}} \mid T^{H_{1}}\right) & =\frac{1}{\zeta(2) i^{2}}
\end{aligned}
$$

where $\zeta$ is the Riemann $\zeta$-function. For the lowest $i$-s, we get:

$$
\begin{aligned}
& \lim _{N \rightarrow \infty} P\left(Y_{1}^{H_{1}} \mid T^{H_{1}}\right) \cong 0,61 \\
& \lim _{N \rightarrow \infty} P\left(Y_{2}^{H_{1}} \mid T^{H_{1}}\right) \cong 0,15 \\
& \lim _{N \rightarrow \infty} P\left(Y_{3}^{H_{1}} \mid T^{H_{1}}\right) \cong 0,068
\end{aligned}
$$


which amounts to an even more pronounced preference of small $i$-s than Menon demands. On this basis, we can see already that the sets of priors demanded by Menon are not entirely unnatural. Rather, they resemble what even a scientist who a priori expects a theory to have infinitely many alternatives might plausibly assume for any empirically viable scientific theory that was developed without guidance by the data that eventually confirmed it.

Note, however, that what is stated in Equations (9) are the posterior probabilities for the $Y_{i}^{H_{1}}$-s of an individual theory $H_{1}$ once its empirical viability has been established. What we are actually interested in are prior probabilities for a theory that has not yet been empirically tested. In order to get there, we need the meta-inductive step of MIA.

\section{A Simplified Take on Meta-Inductive Rea- soning}

A simple approximate way of modeling meta-inductive inference relies on the (false) assumption that each theory that satisfies $K$ has the same number of possible alternatives $i_{K}$. We thus treat the predictive success or failure of each new theory that satisfies $K$ as one more data point for specifying the universal characteristic number $i_{K}$. The modeled process resembles picking balls from one urn with throwing the ball back into the urn after each pick. ${ }^{6}$ Our posterior after the previous pick can serve right away as our prior for the next pick. We can thus simply use the set of posteriors $Y_{i}^{H_{1}}$ that was extracted after $H_{1}$ had been empirically tested as our set of priors $Y_{i}^{H_{2}}$ for the next theory $\mathrm{H}_{2}$ that also meets conditions $K$. We then update on the predictive success or failure of $\mathrm{H}_{2}$ and so on in order to estimate the set of priors $P\left(Y_{i}^{K}\right)$ for all theories that fulfill conditions $K$.

Based on this simple model, it is possible to put the choice of priors chosen in Equation (5) into a wider context. Let us assume a theory $H_{0}$ that satisfies conditions $K$ and start this time with a fully generic distribution of priors $Y_{i}^{H_{0}}$ that has no bias towards low $i$-s:

$$
\begin{array}{rrr}
P\left(Y_{i}^{K}\right)=1 / N & \forall & i \leq N \\
P\left(Y_{i}^{K}\right)=0 & \forall & i>N .
\end{array}
$$

If we update under $T^{H_{0}}$, Equations $(10),(4),(6)$ (all written for $H_{0}$ ), give

\footnotetext{
${ }^{6} \mathrm{We}$ 'd have one white ball in the urn, corresponding to a given theory's predictive success, while all other balls (if there are any) are black, corresponding to predictive failure.
} 


$$
\begin{array}{rrr}
P\left(Y_{i}^{K} \mid T^{H_{0}}\right)=1 /\left(\sum_{j=1}^{N} j^{-1}\right) i & \forall & i \leq N \\
P\left(Y_{i}^{K} \mid T^{H_{0}}\right)=0 \quad & \forall \quad i>N .
\end{array}
$$

which are exactly the priors we assumed in Equation (5). In other words, the probabilities in Equation (5) represent updating on one instance of predictive success from the generic priors of Equations (10). Based on Equations (4) and (6), Equation (11) translates into the following probability of predictive success:

$$
P\left(T^{H_{1}}\right)=\zeta(2) /\left(\sum_{j=1}^{N} j^{-1}\right)
$$

that goes to zero in the $N \rightarrow \infty$ limit.

A second instance of predictive success would then lead to the set of probabilities stated in Equation (8). The analysis suggests that, even with a fully generic set of priors that does not favor individual low values of $i$ at all, two instances of predictive success lead to very substantial values for the smallest $i$-s.

The analysis in this subsection was based on the simplified assumption that all theories that satisfy $K$ have the same number of possible alternatives. The next question will be to what extent the results found in our simplified model remain qualitatively correct in a more adequate model.

\section{Updating Probabilities of Predictive Suc- cess}

Theories satisfying conditions $K$ that address different scientific problems must be expected to have different numbers of possible alternatives. In this light, the probability structure needed for fully characterizing the mechanism of MIA is fairly complex. For each known theory $H_{n}$ that satisfies conditions $K$, there is a true number $i_{n}$ of possible alternatives. We don't know the true $i_{n}$-s. Therefore, we would need to introduce a set of priors $P\left(Y_{i_{n}}\right)$ for each of the s known theories $H_{n}, n=1 \ldots s$ that satisfy conditions $K$. Specifying those sets of priors would give a spectrum of sets of priors. Assuming once again Equation (4), each such spectrum corresponds to a probability of predictive success for a random pick from the theories $H_{n}$. 
Since the only observation-based number that enters MIA is the overall rate of success of theories $H_{n}$, however, we have no observational basis for specifying or updating all dimensions of the spectrum of sets of priors. In order to characterize the situation in a way that can be fully related to empirical data, we thus need to define new variables. One way of doing so is to group the spectra of $i_{n}$ in equivalence classes: We characterize each equivalence class by a real number $I_{K}$ that is defined as

$$
I_{K}^{-1}=\left(\sum_{n=1}^{s}\left(i_{n}^{-1}\right)\right) / s .
$$

$I_{K}^{-1}$ denotes the chances of predictive success of a random-picked theory that satisfies conditions $K$. The equivalence class $I_{K}$ denotes those spectra of numbers of alternatives for theories the meet conditions $\mathrm{K}$ that imply a probability $I_{K}^{-1}$ of predictive success for a randomly picked theory that satisfies conditions $K$. To simplify the calculation, we now take the limit $s \rightarrow \infty$, which turns $I_{K}$ into a continuous function. We are now ready to calculate the Bayesian updating of $\left.P\left(T^{H}\right)\right),{ }^{7}$ starting from the set of priors that corresponds to Equation (10) in an $I_{K}$-based formulation ${ }^{8}$ :

$$
\begin{array}{rrr}
P\left(Y_{I}^{K}\right)=1 / N & \forall & I \leq N+1 \\
P\left(Y_{I}^{K}\right)=0 & \forall & I>N+1 .
\end{array}
$$

In analogy to Equation (4), we assume for any empirically viable but unconfirmed individual theory $\mathrm{H}$ that $\left.P\left(T^{H_{n}}\right) \mid I_{K}\right)=1 / I_{K}$. This implies based on the rule of total probability:

$$
P\left(T^{H_{0}}\right)=(1 / N) \ln (N+1)
$$

which goes to zero in the $N \rightarrow \infty$ limit. Now we carry out Bayesian updating based on two successive instances of predictively successful theories $T^{H_{0}}$ and $T^{H_{1}}$. Taking $T^{H_{0}}$ into account gives:

${ }^{7} H$ denotes a new, so far untested theory whose chances of success we aim to assess, while $H_{0}, H_{1}, \ldots H_{s}$ denotes the series of theories that satisfy conditions $K$ and have been tested already.

${ }^{8}$ Note that this choice of generic priors is not entirely innocuous. For example, the scenario discussed in Section 6 that enforced the same $\mathrm{i}$ for all theories that satisfy $\mathrm{K}$ is a special case of the scenario discussed in Section 7 but is inconsistent with the set set of priors we now specify. 


$$
\begin{aligned}
P\left(Y_{I}^{K} \mid T^{H_{0}}\right) & =\left(\ln \left((N+1) I_{K}\right)\right)^{-1} \\
\left.P\left(T^{H}\right) \mid T^{H_{0}}\right) & =(\ln (N+1))^{-1}
\end{aligned}
$$

This is the continuous form of Equation (12). Like in the former case, $P(T)$ approaches zero in the $N \rightarrow \infty$ limit. Updating under $T^{H_{1}}$ gives

$$
\begin{aligned}
P\left(I_{K} \mid T^{H_{0}}, T^{H_{1}}\right) & =I_{K}^{-2} \\
\left.P\left(T^{H}\right) \mid T^{H_{0}}, T^{H_{1}}\right) & =1 / 2+1 /(2(N+1))
\end{aligned}
$$

We therefore find that two consecutive success stories of scientific theories in the $N, s \rightarrow \infty$ limit lead to a $50 \%$ probability of empirical success for the next theory that satisfies conditions K. High values for low $I_{K}$ and therefore high values for $P\left(T^{H}\right)$ ) are reached quickly from generic priors.

If we carry out further testing of new theories that satisfy $K$, a theory's predictive success will shift probabilities further towards smaller $I_{K^{-}}$, , while a theory's predictive failure will shift probabilities towards higher $I_{K}$-s. The probability, extracted on that basis, for predictive success of the next theory that satisfies $K$ would converge to the success rate one finds among the theories that satisfy $K$ for high numbers of tested theories.

We now see that the first step of updating in the simplified analysis of the previous subsection, that has led to Equation (11), does represent the probabilistic features of the full model. This is not surprising, since, based on the first step of updating, no specific values for $i$ get significant values at all, which means that the question whether any such values indicate the location of the one true value $i_{K}$ or rather a spectrum of true values $i$ for different theories plays no role.

The second updating from Equation (11) to Equation (8) would give an inadequate picture, however, if taken to characterize our credence about the entire set of all theories that satisfy $K$. One can see this clearly if one calculates the probability of predictive success that would correspond to using the set of probabilities of Equation (8) as a set of priors for a new theory $H$. Using once again Equation (4), one would get 0,73, as opposed to the value of $P\left(T^{H}\right)=0,5$ we extracted above. The step towards Equation (8) therefore can only be understood as an updating of the probabilities for $Y_{i}^{H_{1}}$ s under the predictive success of $H_{1}$ but must not be understood as updating of probabilities for $Y_{i}^{H}$-s of a new theory $H$ that satisfies conditions $K$ but has not yet been empirically tested.

The failure of the simplified assumption of one $i_{K}$ for all theories in the given context is to be expected since we are now dealing with results that 
attribute substantial probabilities to individual $i$-s. The question whether our probability distribution represents estimates of one universal $i_{K}$ that characterizes all theories satisfying $K$ or not therefore becomes important. The crucial role of that question is most obvious once one considers updating on a theory's predictive failure. If all theories satisfying $K$ had the same number $i_{K}$ of alternatives, one such failure would send $P\left(Y_{1}\right)$ to zero for all theories that satisfy $K$, which is entirely implausible: the fact that one theory that satisfies $K$ does have alternatives does not rule out the possibility that other theories that address different scientific problems have no alternatives. Only the more complex analysis sketched in this section can account for this fact.

The simplified assumption of one universal $i_{K}$ implies that the expected chances of predictive success of theories that satisfy $K$ cannot rise above $50 \%$ after one instance of predicitive failure. A research field with a success rate of theories that satisfy $K$ significantly above $50 \%$ thus could not be well represented by a probability spectrum $P\left(Y_{i_{K}}\right)$. Any success rate significantly above $50 \%$ thus empirically establishes a multitude of $i$-s among the theories considered.

Let us take stock of where we are at this point. We showed that a theory's predictive success has a very powerful effect on assessing the number of alternatives to that theory: it can generate high probabilities for no or very few alternatives even when starting with the expectation that the number of alternatives is infinite. We then saw that a simplified model of updating in a MIA framework (that is, of updating under the success of other theories that satisfy conditions $K$ ) leads to a strong preference of small numbers of alternatives based on just two instances of predictive success even when starting from priors that do not favor smaller or finite numbers of alternatives at all. Finally, we analyzed a full account of the probabilistic structure of MIA type updating. This did not allow for the extraction of a specific set of posteriors for $Y_{i}$-s. It demonstrated, however, that, while the the simplified calculation of high values for small $Y_{i \text {-s }}$ for a new theory $H$ had to differ significantly from any probability spectrum that could be consistently extracted from a full account of MIA updating, the general conclusion that high probabilities for the lowest priors are reached after two instances of predictive success remains intact.

All three levels of analysis consistently indicate that a pretty focussed Menon-type distribution of $Y_{i}^{H}$-s can be plausibly assumed in a successful research field where several instances of predictive success are known and a tendency of predictive success can be observed. 


\section{MIA and NAA}

The results of the previous section bring us to the second question raised by Menon's analysis. If MIA is so successful on its own in generating high probabilities of predictive success for upcoming as yet empirically untested theories, why do we need NAA at all as an independent argument?

Up to this point, we have painted a fairly idealized picture of MIA. We have assumed that conditions $K$ provide the basis for unequivocally and exhaustively identifying the theories that satisfy conditions $K$. While predictive failures may happen, theories that satisfy $K$ would have a fairly high predictive success rate. This high success rate would then directly inform our credence in the success of the next, so far empirically untested theory that satisfies conditions $K$.

The real world is more messy than that. In the real world, each individual theory we are considering will raise issues as to whether or not it satisfies conditions $K$. The number of theories that satisfy $K$ in a fully convincing way may in the end be fairly small in a given context and individual scientists' assessments regarding the numbers of predictively successful and predictively unsuccessful theories that satisfy $K$ may well differ from each other.

Moreover, each step towards a new theory $H$ that satsfies $K$ raises the question whether the new theory is indeed sufficiently similar in all relevant respects to the previous ones to justify an inference from the the average $Y_{i}$ spectrum we have inferred for theories that satisfy $K$ to the $Y_{i}{ }^{H}$-s for the new theory. Even if one comes to agree that the specified conditions $K$ are met by the given theory, one might suspect that the theory at hand is in relevant respects very different from previous theories that satisfied $K$.

All these considerations will significantly decrease credences for low $i$-s for the new empirically unconfirmed theory compared to the averaged spectrum of $Y_{i}$-probabilities for theories that satisfy $K$. The power of MIA, though arguably quite substantial in successful research fields, therefore must remain limited. It may provide a basis for a substantial preference of low values of $i$. But, on its own, this preference would be too weak for generating a high degree of trust in a new so far empirically untested theory $H$.

This is the reason why NAA is dependent on a convincing case of MIA but nevertheless has a distinct and important role to play. NAA has the advantage over MIA that it is based on looking at one individual theory rather than a spectrum of fairly different theories for which a common denominator must be found. Therefore, it is possible to put particular emphasis on the search for alternatives and the conceptual understanding of the obstacles to such alternatives specifically in cases where the given theory is clearly very different from others satisfying $K$ (Obviously, this characterization fully applies 
to the case of string theory). Future research may be expected to substantially sharpen the understanding regarding NAA, while it would normally not contribute to a further improvement of MIA. In this light, disregarding the independent role of NAA would lead to an inadequate understanding of the ongoing process of scientific theory assessment.

The following overall scenario emerges. MIA can support the significance of NAA type reasoning in a research field by looking at comparable cases where empirical viability could eventually be checked. However, the significance of MIA is constrained by the fact that it is a generalization argument that relies on a set of theories that, though in a relevant sense comparable to the theory we want to confirm, differ from that theory in many other respects. NAA can now step in and rely on investigating the strength of a no alternatives verdict specifically in the given case, carefully searching for alternatives, developing a better theoretical understanding of the obstacles to such attempts, etc. This individual view on the specific context under scrutiny can then, based on the set of priors extracted from MIA, lead to further significant confirmation of the given theory.

To conclude, we find that the $Y_{i}$ distribution needed for a significant NAA doesn't have to be gerrymandered but can be naturally generated by MIA in a predictively successful research field. But due to the limited effectiveness of MIA, NAA is still necessary as an independent argument in order to generate a high level of trust in a theory based on MEC.

\section{Acknowledgements}

I'm very grateful to Stephan Hartmann, Casey McCoy and Tushar Menon for very helpful discussion and remarks on draft versions of this paper. Funding for this research was provided by the Swedish Research Council (project number 1598801).

\section{References}

Dawid, R. (2006): Underdetermination and Theory Succession from the Perspective of String Theory, Philosophy of Science 73/3, 298-322.

Dawid, R. (2013): String Theory and the Scientific Method, Cambridge: Cambridge University Press.

Dawid, R. (2020): Meta-Empirical Confirmation: Addressing Three Points of Criticism, forthcoming. 
Dawid, R. S. Hartmann and Jan Sprenger (2015): "The No Alternatives Argument", The British Journal for the Philosophy of Science 66(1), 213-234.

Menon, T. (2019): "On the Viability of the No Alternatives Argument", Studies in History and Philosophy of Science A, 76:69-75. 